\title{
Inequalities for the Derivative of Rational Functions with Prescribed Poles
}

\author{
Nuttapong Arunrat and Keaitsuda Maneeruk Nakprasit $[$ [ \\ Department of Mathematics, Faculty of Science, Khon Kaen University, Khon Kaen 40002, Thailand \\ Correspondence should be addressed to Keaitsuda Maneeruk Nakprasit; kmaneeruk@hotmail.com
}

Received 23 June 2020; Accepted 17 August 2020; Published 15 September 2020

Academic Editor: Teodor Bulboaca

Copyright ( $\odot 2020$ Nuttapong Arunrat and Keaitsuda Maneeruk Nakprasit. This is an open access article distributed under the Creative Commons Attribution License, which permits unrestricted use, distribution, and reproduction in any medium, provided the original work is properly cited.

In this paper, we consider a class of rational functions $r(s(z))$ of degree $m n$ where $s(z)$ is a polynomial of degree $m$ and establish some inequalities for rational functions with prescribed poles which generalize and refine the result of I. Qasim and A. Liman.

\section{Introduction}

Let $P_{n}$ denote the class of all complex polynomials of degree at most $n$ and let $k$ be a positive real number. We denote $T_{k}=\{z:|z|=k\},\left\{D_{k-}=z:|z|<k\right\}$, and $D_{k+}=\{z:|z|>k\}$. Consider a polynomial $p(z)$ of degree $n$. In 1926, Bernstein [1] presented the following well-known inequality:

$$
\max _{|z|=1}\left|p^{\prime}(z)\right| \leq n \max _{|z|=1}|p(z)|
$$

Equality holds in (1) only for $p(z)=a z^{n}$, where $a \neq 0$. If we restrict to the class of polynomials having no zeros in $D_{1-}$, inequality (1) can be sharpened. In fact, it was conjectured by P. Erdö s and later proved by Lax [2] that if $p(z)$ has no zeros in $D_{1-}$, then

$$
\max _{|z|=1}\left|p^{\prime}(z)\right| \leq \frac{n}{2} \max _{|z|=1}|p(z)|
$$

For the class of polynomials having no zeros in $D_{1+}$, Turán [3] proved that

$$
\max _{|z|=1}\left|p^{\prime}(z)\right| \geq \frac{n}{2} \max _{|z|=1}|p(z)| .
$$

For $a_{j} \in \mathbb{C}(1 \leq j \leq n)$, we let $w(z)=\prod_{j=1}^{n}\left(z-a_{j}\right)$ and

$$
\begin{aligned}
B(z) & =\prod_{j=1}^{n}\left(\frac{1-\overline{a_{j}} z}{z-a_{j}}\right), \\
R_{n} & =R_{n}\left(a_{1}, a_{2}, \ldots, a_{n}\right):=\left\{\frac{p(z)}{w(z)}: p \in P_{n}\right\} .
\end{aligned}
$$

The product $B(z)$ is known as a Blaschke product.

Then, $R_{n}$ is the set of rational functions with at most $n$ poles $a_{1}, a_{2}, \ldots, a_{n}$ and with finite limit at infinity. For $f$ defined on $T_{1}$, we denote $\|f\|=\sup _{z \in T_{1}}|f(z)|$, the Chebyshev norm of $f$ on $T_{1}$. Throughout this paper, we assume that all poles $a_{1}, a_{2}, \ldots, a_{n}$ are in $D_{1+}$.

In 1995, Li et al. [4] proved some inequalities similar to (1), (2), and (3) for rational functions. Among other things, they proved the following result.

Theorem 1 (see [4]). Let $r \in R_{n}$ with all its zeros lying in $T_{1} \cup D_{1+}$. Then, for $z \in T_{1}$,

$$
\left|r^{\prime}(z)\right| \leq \frac{1}{2}\left|B^{\prime}(z)\right| \cdot\|r\|
$$

Equality holds for $r(z)=a B(z)+b$ with $|a|=|b|=1$.

In 1997, inequality (5) was improved by Aziz and Shah [5] under the same hypothesis. They obtained the following theorem. 
Theorem 2 (see [5]). Let $r \in R_{n}$ with all its zeros lying in $T_{1} \cup D_{1+}$. Then, for $z \in T_{1}$,

$$
\left|r^{\prime}(z)\right| \leq \frac{1}{2}\left|B^{\prime}(z)\right|(\|r\|-m),
$$

where $m=\min _{|z|=1}|r(z)|$. Equality holds for $r(z)=B(z)+$ $h e^{i \alpha}$ where $h \geq 1$ and $\alpha$ is real.

In 1999, Aziz and Zarger [6] considered a class of rational functions $R_{n}$ not vanishing in $T_{k} \cup D_{k-}$, where $k \geq 1$, and established the following generalization of Theorem 1 .

Theorem 3 (see [6]). Let $r \in R_{n}$ with all its zeros lying in $T_{k} \cup D_{k+}$, where $k \geq 1$. Then, for $z \in T_{1}$,

$$
\left|r^{\prime}(z)\right| \leq \frac{1}{2}\left[\left|B^{\prime}(z)\right|-\frac{n(k-1)}{k+1} \cdot \frac{|r(z)|^{2}}{\|r\|^{2}}\right] \cdot\|r\| .
$$

Equality holds for $r(z)=((z+k) /(z-a))^{n}$ and $B(z)=$ $((1-a z) /(z-a))^{n}$ evaluated at $z=1$, where $a>1$ and $k \geq 1$.

Recently, inequalities (6) and (7) were improved by Arunrat and Nakprasit [7] under the same hypothesis. They obtained the following theorem.

Theorem 4 (see [7]). Let $r \in R_{n}$, where $r$ has exactly $n$ poles at $a_{1}, a_{2}, \ldots, a_{n}$ and all its zeros lie in $T_{k} \cup D_{k+}, k \geq 1$. Then, for $z \in T_{1}$,

$$
\left|r^{\prime}(z)\right| \leq \frac{1}{2}\left[\left|B^{\prime}(z)\right|-\frac{(n(1+k)-2 t)(|r(z)|-m)^{2}}{(1+k)(\|r\|-m)^{2}}\right](\|r\|-m),
$$

where $t$ is the number of zeros of $r$ with counting multiplicity and $m=\min _{|z|=k}|r(z)|$. Equality holds for $r(z)=((z+$ $\left.k)^{t} /(z-a)^{n}\right)$ and $B(z)=((1-a z) /(z-a))^{n}$ evaluated at $z=1, a>1$, and $k \geq 1$.

In 2015, Qasim and Liman [8] considered a class of rational functions $r(s(z)) \in R_{m n}$ with all poles $a_{1}, a_{2}, \ldots, a_{m n}$ lying in $D_{1+}$, defined by

$$
(r \circ s)(z)=r(s(z)):=\frac{p(s(z))}{w(s(z))}
$$

where $s(z)$ is a polynomial of degree $m$ with all its zeros lying in $T_{1} \cup D_{1-}$ and $r \in R_{n}$. Let

$$
w(s(z))=\prod_{j=1}^{m n}\left(z-a_{j}\right)
$$

and the Blaschke product

$$
B(z)=\frac{w^{*}(s(z))}{w(s(z))}=\frac{z^{m n} \overline{w(s(1 / \bar{z}))}}{w(s(z))}=\prod_{j=1}^{m n}\left(\frac{1-\overline{a_{j}} z}{z-a_{j}}\right) .
$$

They proved the following generalization of inequality (5).

Theorem 5. (see [8]). Let $r(s(z)) \in R_{m n}$, where $r(s(z))$ has no zeros in $D_{1-}$ and all zeros of $s(z)$ lie in $T_{1} \cup D_{1-}$. Then, for $z \in T_{1}$,

$$
\left|r^{\prime}(s(z))\right| \leq \frac{1}{2 m m^{\prime}}\left|B^{\prime}(z)\right| \cdot\|r \circ s\|
$$

where $m^{\prime}=\min _{z \in T_{1}}|s(z)|$. The inequality is sharp and equality holds for $r(s(z))=a B(z)+b$ with $a, b \in T_{1}$ and $s(z)=z^{m}$.

Observe that if $s(z)$ has a zero on $T_{1}$, then $m^{\prime}=0$, and we obtain a trivial inequality:

$$
0=2 m m^{\prime} \cdot\left|r^{\prime}(s(z))\right| \leq\left|B^{\prime}(z)\right|\|r \circ s\| .
$$

In this paper, we consider the class of rational functions $R_{m n}$ having no zeros in $D_{k-}$, where $k \geq 1$, and prove the generalization of the result of Qasim and Liman [8].

\section{Lemmas}

For the proof of our main theorems, we need the following lemmas. These two lemmas are due to Li et al. [4].

Lemma 1 (see [4]). Let $r \in R_{n}$. If all zeros of $r$ lie in $T_{1} \cup D_{1+}$, then, for $z \in T_{1}$,

$$
\operatorname{Re}\left(\frac{z r^{\prime}(z)}{r(z)}\right) \leq \frac{\left|B^{\prime}(z)\right|}{2}
$$

where $r(z) \neq 0$.

Lemma 2 (see [4]). If $r \in R_{n}$ and $r^{*}(z)=B(z) \overline{r(1 / \bar{z})}$, then, for $z \in T_{1}$,

$$
\left|\left(r^{*}(z)\right)^{\prime}\right|+\left|r^{\prime}(z)\right| \leq\left|B^{\prime}(z)\right| \cdot\|r\| .
$$

Equality holds for $r(z)=a B(z)$ with $a \in T_{1}$.

Lemma 3 is due to Aziz and Dawood [9].

Lemma 3 (see [9]). If $p \in P_{n}$ and $p(z)$ has all its zeros in $T_{1} \cup D_{1-}$, then

$$
\min _{z \in T_{1}}\left|p^{\prime}(z)\right| \geq n \cdot \min _{z \in T_{1}}|p(z)| .
$$

The inequality is sharp and equality holds for polynomials having all zeros at the origin.

Lemma 4 is due to Aziz and Shah [5], and Lemma 5 is due to Arunrat and Nakprasit [7].

Lemma 4 (see [5]). If $B(z)$ is Blaschke product and $\alpha$ is real, $0 \leq \alpha<2 \pi$, then $B(z)+h e^{i \alpha}$ has all its zeros in $T_{1} \cup D_{1+}$, for every $h \geq 1$.

Lemma 5 (see [7]). Assume that $r \in R_{n}$, where $r$ has exactlyn poles at $a_{1}, a_{2}, \ldots, a_{n}$. Let $t$ be the number of zeros of $r$ with counting multiplicity. If all zeros of $r$ lie in $T_{k} \cup D_{k+}$, where $k \geq 1$, and $z \in T_{1}$ with $r(z) \neq 0$, then

$$
\operatorname{Re}\left(\frac{z r^{\prime}(z)}{r(z)}\right) \leq \frac{\left|B^{\prime}(z)\right|}{2}+\frac{2 t-n(1+k)}{2(1+k)} \text {. }
$$




\section{Main Theorems}

In this section, we state and prove main results. One of them generalizes the result of Qasim and Liman [8].

Theorem 6. Let $r(s(z)) \in R_{m n}$ with $r(s(z)) \neq 0$ in $D_{1-}$ and all zeros of $s(z)$ lie in $T_{1} \cup D_{1-}$. Then, for $z \in T_{1}$,

$$
\left|r^{\prime}(s(z))\right| \leq \frac{1}{2 m m^{\prime}}\left|B^{\prime}(z)\right| \cdot\left(\|r \circ s\|-m^{*}\right),
$$

where $m^{\prime}=\min _{z \in T_{1}}|s(z)|$ and $m^{*}=\min _{z \in T_{1}}|r(s(z))|$.

Equality holds for $r(s(z))=B(z)+h e^{i \alpha}$ where $s(z)=z^{m}, h \geq 1$, and $\alpha$ is real.

Proof. Let $r(s(z)) \in R_{m n}$ without zeros in $|z|<1$ and $m^{*}=\min _{z \in T_{1}}|r(s(z))|$.

Therefore, $m^{*} \leq|r(s(z))|$ for $z \in T_{1}$. If $r(s(z))$ has a zero on $T_{1}$, then $m^{*}=0$, and hence, for every $\alpha$ with $|\alpha|<1$, we get that $r(s(z))-\alpha m^{*}=r(s(z))$. In case $r(s(z))$ has no zeros on $T_{1}$, we have for every $\alpha$ with $|\alpha|<1$ that $\left|-\alpha m^{*}\right|=$ $|\alpha| \cdot m^{*}<|r(s(z))|$ for $|z|=1$. It follows from Rouche's theorem that rational functions $R(z)=r(s(z))-\alpha m^{*}$ and $r(s(z))$ have the same number of zeros in $D_{1-}$. That is, for every $\alpha$ with $|\alpha|<1, R(z)$ has no zeros in $D_{1-}$. We first assume that $R(z) \neq 0$. Lemma 1 yields that for $z \in T_{1}$,

$$
\operatorname{Re}\left(\frac{z R^{\prime}(z)}{R(z)}\right) \leq \frac{\left|B^{\prime}(z)\right|}{2} .
$$

Let $\quad R^{*}(z)=B(z) \overline{R(1 / \bar{z})}=B(z) \bar{R}(1 / z) . \quad$ Then, $\left(R^{*}(z)\right)^{\prime}=B^{\prime}(z) \bar{R}(1 / z)-\left(B(z) / z^{2}\right) \cdot \bar{R}^{\prime}(1 / z)$.

Consequently, $z\left(R^{*}(z)\right)^{\prime}=z B^{\prime}(z) \bar{R}(1 / z)-(B(z) / z) \cdot \bar{R}^{\prime}(1 / z)$.

Since $z \in T_{1}, \quad$ we have $\bar{z}=(1 / z), \quad|B(z)|=1$, $\left(\left(z B^{\prime}(z)\right) /(B(z))\right)=\left|B^{\prime}(z)\right|$, and so

$$
\left|z\left(R^{*}(z)\right)^{\prime}\right|=\left|z B^{\prime}(z) \overline{R(z)}-B(z) \overline{z R^{\prime}(z)}\right|=\left|\frac{z B^{\prime}(z)}{B(z)} \cdot \overline{R(z)}-\overline{z R^{\prime}(z)}\right|=|| B^{\prime}(z)\left|\overline{R(z)}-\overline{z R^{\prime}(z)}\right| .
$$

Since $\left|B^{\prime}(z)\right|$ is real, we obtain that Then, $\left|z\left(R^{*}(z)\right)^{\prime}\right|=\| B^{\prime}(z)\left|R(z)-z R^{\prime}(z)\right|$.

$$
\begin{aligned}
\left|\frac{z\left(R^{*}(z)\right)^{\prime}}{R(z)}\right|^{2}= & || B^{\prime}(z)\left|-\frac{z R^{\prime}(z)}{R(z)}\right|^{2}=\left|B^{\prime}(z)\right|^{2}-2\left|B^{\prime}(z)\right| \cdot \operatorname{Re}\left(\frac{z R^{\prime}(z)}{R(z)}\right)+\left|\frac{z R^{\prime}(z)}{R(z)}\right|^{2} \geq\left|B^{\prime}(z)\right|^{2}-2\left|B^{\prime}(z)\right|\left(\frac{\left|B^{\prime}(z)\right|}{2}\right) \\
& +\left|\frac{z R^{\prime}(z)}{R(z)}\right|^{2}=\left|\frac{z R^{\prime}(z)}{R(z)}\right|^{2},
\end{aligned}
$$

where the inequality comes from (19).

This implies that for $z \in T_{1}$ which are not the zeros of $R(z)$,

$$
\left|R^{\prime}(z)\right| \leq\left|\left(R^{*}(z)\right)^{\prime}\right|
$$

where $\quad R^{*}(z)=B(z) \overline{R(1 / \bar{z})}=r^{*}(s(z))-\bar{\alpha} m^{*} B(z) \quad$ with $r^{*}(s(z))=B(z) \overline{r(s(1 / \bar{z}))}$.

Moreover, $\left(R^{*}(z)\right)^{\prime}=\left(r^{*}(s(z))\right)^{\prime}-\bar{\alpha} m^{*} B^{\prime}(z) \quad$ and $R^{\prime}(z)=(r(s(z)))^{\prime}$.

Applying these relations into (22), we obtain that

$$
\left|(r(s(z)))^{\prime}\right| \leq\left|\left(r^{*}(s(z))\right)^{\prime}-\bar{\alpha} m^{*} B^{\prime}(z)\right|,
$$

for $z \in T_{1}$ with $R(z) \neq 0$ and every $\alpha$ with $|\alpha|<1$.

Choose the argument of $\alpha$ so that

$$
\left|\left(r^{*}(s(z))\right)^{\prime}-\bar{\alpha} m^{*} B^{\prime}(z)\right|=\left|\left(r^{*}(s(z))\right)^{\prime}\right|-m^{*}|\alpha|\left|B^{\prime}(z)\right|,
$$

for $z \in T_{1}$ with $R(z) \neq 0$.

Substituting relation (24) into (23), we obtain that

$$
\left|(r(s(z)))^{\prime}\right| \leq\left|\left(r^{*}(s(z))\right)^{\prime}\right|-m^{*}|\alpha|\left|B^{\prime}(z)\right| .
$$

Letting $|\alpha| \longrightarrow 1$, we obtain

$$
\left|(r(s(z)))^{\prime}\right| \leq\left|\left(r^{*}(s(z))\right)^{\prime}\right|-m^{*}\left|B^{\prime}(z)\right|
$$

Lemma 2 implies that

$\left|(r(s(z)))^{\prime}\right| \leq\left|B^{\prime}(z)\right| \cdot\|r \circ s\|-\left|(r(s(z)))^{\prime}\right|-m^{*}\left|B^{\prime}(z)\right|$.

Thus,

$$
\left|(r(s(z)))^{\prime}\right| \leq \frac{1}{2}\left|B^{\prime}(z)\right| \cdot\left(\|r \circ s\|-m^{*}\right) .
$$

For $z \in T_{1} \quad$ with $\quad R(z) \neq 0$, we have $\left|(r(s(z)))^{\prime}\right| \geq\left|r^{\prime}(s(z))\right| \cdot \min _{z \in T_{1}}\left|s^{\prime}(z)\right|$.

From Lemma 3, we obtain that

$$
\left|(r(s(z)))^{\prime}\right| \geq\left|r^{\prime}(s(z))\right| \cdot\left(m \cdot \min _{z \in T_{1}}|s(z)|\right)=m m^{\prime}\left|r^{\prime}(s(z))\right| .
$$

It follows from (28) that

$$
\left|r^{\prime}(s(z))\right| \leq \frac{1}{2 m m^{\prime}}\left|B^{\prime}(z)\right| \cdot\left(\|r \circ s\|-m^{*}\right) .
$$

This proves inequality for $R(z) \neq 0$. In case $R(z)=0$, we obtain that $(r(s(z)))^{\prime}=0$. 
This implies that the above inequality is trivially true.

Therefore, inequality (18) holds for all $z \in T_{1}$.

Next, we show that equality holds for $r(s(z))=B(z)+$ $h e^{i \alpha}$ where $h \geq 1$ and $s(z)=z^{m}$. Lemma 4 implies that $B(z)+$ $h e^{i \alpha}$ has all its zeros in $T_{1} \cup D_{1+}$. Moreover, we obtain that

$$
\begin{aligned}
\|r \circ s\| & =\max _{z \in T_{1}}\left|B(z)+h e^{i \alpha}\right|=h+1, \\
m^{*} & =\min _{z \in T_{1}}\left|B(z)+h e^{i \alpha}\right|=h-1, \\
m^{\prime} & =\min _{z \in T_{1}}|s(z)|=1 .
\end{aligned}
$$

Consider $B^{\prime}(z)=(r(s(z)))^{\prime}=r^{\prime}(s(z)) \cdot s^{\prime}(z)=m z^{m-1} \cdot r^{\prime}(s(z))$.

This implies that $r^{\prime}(s(z))=\left(B^{\prime}(z) / m z^{m-1}\right)$. Then, for $z \in T_{1},\left|r^{\prime}(s(z))\right|=\left(\left|B^{\prime}(z)\right| / m\right)$.

The right side of inequality (18) is

$$
\frac{1}{2 m m^{\prime}}\left|B^{\prime}(z)\right| \cdot\left(\|r \circ s\|-m^{*}\right)=\frac{1}{2 m(1)}\left|B^{\prime}(z)\right| \cdot((h+1)-(h-1))=\left|r^{\prime}(s(z))\right| .
$$

Thus, this bound is best possible.

Theorem 7. Let $r(s(z)) \in R_{m n}$ with $r(s(z)) \neq 0$ in $D_{k-}, k \geq 1$, and all zeros of $s(z)$ lie in $T_{1} \cup D_{1-}$. Then, for $z \in T_{1}$,

$$
\left|r^{\prime}(s(z))\right| \leq \frac{1}{2 m m^{\prime}}\left[\left|B^{\prime}(z)\right|-\frac{(m n(1+k)-2 m t)\left(|r(s(z))|-m^{*}\right)^{2}}{(1+k)\left(\|r \circ s\|-m^{*}\right)^{2}}\right]\left(\|r \circ s\|-m^{*}\right),
$$

where $m t$ is the number of zeros of $r \circ s$ with counting multiplicity, $m^{\prime}=\min _{z \in T_{1}}|s(z)|$, and $m^{*}=\min _{z \in T_{k}}|r(s(z))|$. Equality holds for $r(s(z))=\left((z+k)^{m t} /(z-a)^{m n}\right)$, where $s(z)=z^{m}$ and $B(z)=((1-a z) /(z-a))^{m n}, a>1$ and $k \geq 1$ at $z=1$.

Proof. Let $r(s(z)) \in R_{m n}$ without zeros in $|z|<k$, where $k \geq 1$.

Let $m^{*}=\min _{z \in T_{k}}|r(s(z))|$ and $m t$ be the number of zeros of $r \circ s$ with counting multiplicity. Therefore, $m^{*} \leq|r(s(z))|$ for $z \in T_{k}$. If $r(s(z))$ has a zero on $T_{k}$, then $m^{*}=0$, and hence, for every $\alpha$ with $|\alpha|<1$, we obtain that $r(s(z))-\alpha m^{*}=r(s(z))$. In case $r(s(z))$ has no zeros on $T_{k}$, we have for every $\alpha$ with $|\alpha|<1$ that $\left|-\alpha m^{*}\right|=|\alpha| \cdot m^{*}<|r(s(z))|$ for $|z|=k$. Therefore, it follows from Rouche's theorem that rational functions $R(z)=r(s(z))-\alpha m^{*}$ and $r(s(z))$ have the same number of zeros in $D_{k-}$. That is, for every $\alpha$ with $|\alpha|<1, R(z)$ has no zeros in $D_{k_{-}}$. We first assume that $R(z) \neq 0$. Lemma 5 yields that for $z \in T_{1}$,

$$
\operatorname{Re}\left(\frac{z R^{\prime}(z)}{R(z)}\right) \leq \frac{\left|B^{\prime}(z)\right|}{2}+\frac{2 m t-m n(1+k)}{2(1+k)}
$$

Let $\quad R^{*}(z)=B(z) \overline{R(1 / \bar{z})}=B(z) \bar{R}(1 / z) . \quad$ Then, $\left(R^{*}(z)\right)^{\prime}=B^{\prime}(z) \bar{R}(1 / z)-\left(B(z) / z^{2}\right) \cdot \bar{R}^{\prime}(1 / z)$.

Consequently, $z\left(R^{*}(z)\right)^{\prime}=z B^{\prime}(z) \bar{R}(1 / z)-(B(z) / z) \cdot \bar{R}^{\prime}(1 / z)$.

Since $z \in T_{1}$, we have $\bar{z}=(1 / z), \quad|B(z)|=1$, $\left(z B^{\prime}(z) / B(z)\right)=\left|B^{\prime}(z)\right|$, and so

$$
\left|z\left(R^{*}(z)\right)^{\prime}\right|=\left|z B^{\prime}(z) \overline{R(z)}-B(z) \overline{z R^{\prime}(z)}\right|=\left|\frac{z B^{\prime}(z)}{B(z)} \cdot \overline{R(z)}-\overline{z R^{\prime}(z)}\right|=|| B^{\prime}(z)\left|\overline{R(z)}-\overline{z R^{\prime}(z)}\right| .
$$

Since $\left|B^{\prime}(z)\right|$ is real, we obtain that Then, $\left|z\left(R^{*}(z)\right)^{\prime}\right|=\| B^{\prime}(z)\left|R(z)-z R^{\prime}(z)\right|$.

$$
\begin{aligned}
\left|\frac{z\left(R^{*}(z)\right)^{\prime}}{R(z)}\right|^{2}= & || B^{\prime}(z)\left|-\frac{z R^{\prime}(z)}{R(z)}\right|^{2}=\left|B^{\prime}(z)\right|^{2}-2\left|B^{\prime}(z)\right| \cdot \operatorname{Re}\left(\frac{z R^{\prime}(z)}{R(z)}\right)+\left|\frac{z R^{\prime}(z)}{R(z)}\right|^{2} \geq\left|B^{\prime}(z)\right|^{2} \\
& -2\left|B^{\prime}(z)\right|\left[\frac{1}{2}\left(\left|B^{\prime}(z)\right|+\frac{2 m t-m n(1+k)}{(1+k)}\right)\right]+\left|\frac{z R^{\prime}(z)}{R(z)}\right|^{2}=\left|\frac{z R^{\prime}(z)}{R(z)}\right|^{2}+\frac{m n(1+k)-2 m t}{(1+k)} \cdot\left|B^{\prime}(z)\right|
\end{aligned}
$$


where the inequality comes from (34).

This implies that for $z \in T_{1}$ which are not zeros of $R(z)$,where $\quad R^{*}(z)=B(z) \overline{R(1 / \bar{z})}=r^{*}(s(z))-\bar{\alpha} m^{*} B(z)$ with $r^{*}(s(z))=B(z) \overline{r(s(1 / \bar{z}))}$,

$$
\left[\left|R^{\prime}(z)\right|^{2}+\frac{m n(1+k)-2 m t}{(1+k)} \cdot|R(z)|^{2}\left|B^{\prime}(z)\right|\right]^{(1 / 2)} \leq\left|\left(R^{*}(z)\right)^{\prime}\right| \text {, }
$$

Moreover, $\quad\left(R^{*}(z)\right)^{\prime}=\left(r^{*}(s(z))\right)^{\prime}-\bar{\alpha} m^{*} B^{\prime}(z) \quad$ and $R^{\prime}(z)=\left(r(s(z))-\alpha m^{*}\right)^{\prime}=(r(s(z)))^{\prime}$.

Applying these relations into (37), we obtain that

$$
\left[\left|(r(s(z)))^{\prime}\right|^{2}+\frac{m n(1+k)-2 m t}{(1+k)} \cdot\left|r(s(z))-\alpha m^{*}\right|^{2}\left|B^{\prime}(z)\right|\right]^{(1 / 2)} \leq\left|\left(r^{*}(s(z))\right)^{\prime}-\bar{\alpha} m^{*} B^{\prime}(z)\right|,
$$

for $z \in T_{1}$ with $R(z) \neq 0$ and every $\alpha$ with $|\alpha|<1$.

Choose the argument of $\alpha$ so that

$$
\left|\left(r^{*}(s(z))\right)^{\prime}-\bar{\alpha} m^{*} B^{\prime}(z)\right|=\left|\left(r^{*}(s(z))\right)^{\prime}\right|-m^{*}|\alpha|\left|B^{\prime}(z)\right| \text {, }
$$

for $z \in T_{1}$ with $R(z) \neq 0$.

Triangle inequality yields that $\left|r(s(z))-\alpha m^{*}\right| \geq \| r$ $(s(z))\left|-m^{*}\right| \alpha||$.
Note that ||$r(s(z))\left|-m^{*}\right| \alpha||^{2}=\left(|r(s(z))|-m^{*}|\alpha|\right)^{2}$ which implies that

$$
\left|r(s(z))-m^{*} \alpha\right|^{2} \geq\left(|r(s(z))|-m^{*}|\alpha|\right)^{2} .
$$
that

Substituting relations (40) and (39) into (38), we obtain

$$
\left[\left|(r(s(z)))^{\prime}\right|^{2}+\frac{m n(1+k)-2 m t}{(1+k)} \cdot\left(|r(s(z))|-m^{*}|\alpha|\right)^{2}\left|B^{\prime}(z)\right|\right]^{(1 / 2)} \leq\left|\left(r^{*}(s(z))\right)^{\prime}\right|-m^{*}|\alpha|\left|B^{\prime}(z)\right| .
$$

Letting $|\alpha| \longrightarrow 1$, we obtain

$$
\left[\left|(r(s(z)))^{\prime}\right|^{2}+\frac{m n(1+k)-2 m t}{(1+k)} \cdot\left(|r(s(z))|-m^{*}\right)^{2}\left|B^{\prime}(z)\right|\right]^{(1 / 2)} \leq\left|\left(r^{*}(s(z))\right)^{\prime}\right|-m^{*}\left|B^{\prime}(z)\right| .
$$

Lemma 2 implies that

$$
\left[\left|(r(s(z)))^{\prime}\right|^{2}+\frac{m n(1+k)-2 m t}{(1+k)} \cdot\left(r(s(z))-m^{*}\right)^{2}\left|B^{\prime}(z)\right|\right]^{(1 / 2)} \leq\left|B^{\prime}(z)\right| \cdot\|r \circ s\|-\left|(r(s(z)))^{\prime}\right|-m^{*}\left|B^{\prime}(z)\right| .
$$

Equivalently,

$$
\left|(r(s(z)))^{\prime}\right|^{2}+\frac{m n(1+k)-2 m t}{(1+k)} \cdot\left(|r(s(z))|-m^{*}\right)^{2}\left|B^{\prime}(z)\right| \leq\left[\left(\|r \circ s\|-m^{*}\right)\left|B^{\prime}(z)\right|-\left|(r(s(z)))^{\prime}\right|\right]^{2} .
$$

Hence,

$$
\begin{gathered}
\left|(r(s(z)))^{\prime}\right|^{2}+\frac{m n(1+k)-2 m t}{(1+k)} \cdot\left(|r(s(z))|-m^{*}\right)^{2}\left|B^{\prime}(z)\right| \leq \\
\left(\|r \circ s\|-m^{*}\right)^{2}\left|B^{\prime}(z)\right|^{2}-2\left(\|r \circ s\|-m^{*}\right)\left|B^{\prime}(z) \|(r(s(z)))^{\prime}\right| \\
+\left|(r(s(z)))^{\prime}\right|^{2} .
\end{gathered}
$$


Then,

$$
2\left(\|r \circ s\|-m^{*}\right)\left|B^{\prime}(z)\right||(r(s(z)))|^{\prime} \leq\left(\|r \circ s\|-m^{*}\right)^{2}\left|B^{\prime}(z)\right|^{2}-\frac{m n(1+k)-2 m t}{(1+k)} \cdot\left(|r(s(z))|-m^{*}\right)^{2}\left|B^{\prime}(z)\right| .
$$

That is,

$$
2\left(\|r \circ s\|-m^{*}\right)|(r(s(z)))|^{\prime} \leq\left(\|r \circ s\|-m^{*}\right)^{2}\left|B^{\prime}(z)\right|-\frac{m n(1+k)-2 m t}{(1+k)} \cdot\left(r(s(z))-m^{*}\right)^{2}
$$

Thus,

$$
\begin{aligned}
& \begin{aligned}
\left|(r(s(z)))^{\prime}\right| & \leq \frac{1}{2}\left[\frac{\left(\|r \circ s\|-m^{*}\right)^{2}\left|B^{\prime}(z)\right|}{\left(\|r \circ s\|-m^{*}\right)}-\frac{(m n(1+k)-2 m t)\left(|r(s(z))|-m^{*}\right)^{2}}{(1+k)\left(\|r \circ s\|-m^{*}\right)}\right] \\
& =\frac{1}{2}\left[\left|B^{\prime}(z)\right|-\frac{\left.(m n(1+k)-2 m t)\left(|r(s(z))|-m^{*}\right)^{2}\right]}{(1+k)\left(\|r \circ s\|-m^{*}\right)^{2}}\right]\left(\|r \circ s\|-m^{*}\right) .
\end{aligned} \\
& R(z) \neq 0, \text { we have } \quad\left|(r(s(z)))^{\prime}\right| \geq\left|r^{\prime}(s(z))\right| \cdot\left(m \cdot \min _{z \in T_{1}}|s(z)|\right) \\
& =m m^{\prime}\left|r^{\prime}(s(z))\right| .
\end{aligned}
$$

$\left|(r(s(z)))^{\prime}\right|=\left|r^{\prime}(s(z)) \cdot s^{\prime}(z)\right|=\left|r^{\prime}(s(z))\right| \cdot\left|s^{\prime}(z)\right|$

$$
\geq\left|r^{\prime}(s(z))\right| \cdot \min _{z \in T_{1}}\left|s^{\prime}(z)\right| \text {. }
$$

Therefore, it follows from (48) that

From Lemma 3, we obtain that

$$
\left|r^{\prime}(s(z))\right| \leq \frac{1}{2 m m^{\prime}}\left[\left|B^{\prime}(z)\right|-\frac{(m n(1+k)-2 m t)\left(|r(s(z))|-m^{*}\right)^{2}}{(1+k)\left(\|r \circ s\|-m^{*}\right)^{2}}\right]\left(\|r \circ s\|-m^{*}\right),
$$

where $m t$ is the number of zeros of $r \circ s$ with counting multiplicity, $m^{\prime}=\min _{z \in T_{1}}|s(z)|$, and $m^{*}=\min _{z \in T_{k}}|r(s(z))|$. This proves inequality for $R(z) \neq 0$.

In case $R(z)=0$, we obtain that $(r(s(z)))^{\prime}=0$.

This implies that the above inequality is trivially true.

Therefore, inequality (33) holds for all $z \in T_{1}$.

Next, we show that equality holds for $r(s(z))=\left((z+k)^{m t} /(z-a)^{m n}\right)$ where $s(z)=z^{m}$ and $B(z)=((1-a z) /(z-a))^{m n}, a>1$, and $k \geq 1$ at $z=1$. First, we observe that $\|r \circ s\|=\left((1+k)^{m t} /(a-1)^{m n}\right)=|r(s(1))|$, $m^{\prime}=1, m^{*}=0,\left|B^{\prime}(1)\right|=(m n(a+1) /(a-1)), \quad$ and

$$
r^{\prime}(s(z))=\left((z+k)^{m t} / m z^{m-1}(z-a)^{m n}\right)[(m t / z+k)+(m n /
$$
$a-z)]$.

Then,

$$
\begin{aligned}
\left|r^{\prime}(s(1))\right| & =\frac{(1+k)^{m t}}{m(1)^{m-1}(a-1)^{m n}}\left[\frac{m t}{1+k}+\frac{m n}{a-1}\right] \\
& =\left[\frac{t}{1+k}+\frac{n}{a-1}\right] \cdot\|r \circ s\| .
\end{aligned}
$$

The right side of inequality (33) is

$$
\begin{aligned}
\frac{1}{2 m m^{\prime}}\left[\left|B^{\prime}(1)\right|-\frac{(m n(1+k)-2 m t)\left(|r(s(1))|-m^{*}\right)^{2}}{(1+k)\left(\|r \circ s\|-m^{*}\right)^{2}}\right]\left(\|r \circ s\|-m^{*}\right) & =\frac{1}{2 m(1)}\left[\frac{m n(a+1)}{a-1}-\frac{(m n(1+k)-2 m t)}{1+k}\right] \cdot\|r \circ s\| \\
& =\frac{1}{2}\left[\frac{2 n}{a-1}+\frac{2 t}{1+k}\right] \cdot\|r \circ s\|=\left|r^{\prime}(s(1))\right| .
\end{aligned}
$$


Thus, this bound is best possible.

Theorem 7 simplifies to Theorem 4 when $s(z)=z$.

Observe that $m n(1+k)-2 m t \geq m n(k-1)$ for all $t \leq n$. We obtain an immediately consequence of Theorem 7 as follows.
Corollary 1. Let $r(s(z)) \in R_{m n}$ with $r(s(z)) \neq 0$ in $D_{k-}, k \geq 1$, and all zeros of $s(z)$ lie in $T_{1} \cup D_{1-}$. Then, for $z \in T_{1}$,

$$
\left|r^{\prime}(s(z))\right| \leq \frac{1}{2 m m^{\prime}}\left[\left|B^{\prime}(z)\right|-\frac{m n(k-1)\left(|r(s(z))|-m^{*}\right)^{2}}{(1+k)\left(\|r \circ s\|-m^{*}\right)^{2}}\right]\left(\|r \circ s\|-m^{*}\right),
$$

where $m^{\prime}=\min _{z \in T_{1}}|s(z)|$ and $m^{*}=\min _{z \in T_{k}}|(r(s(z)))|$. Equality holds for $r(s(z))=((z+k) /(z-a))^{m n}$ where $s(z)=z^{m}$ and $B(z)=((1-a z) /(z-a))^{m n}, a>1$, and $k \geq 1$ at $z=1$.

From Corollary 1, if $r(s(z))$ has all its zeros in $T_{k} \cup D_{k+}$ with at least one zero on $T_{k}$, we obtain the following corollary.

Corollary 2. Let $r(s(z)) \in R_{m n}$ and $r(s(z)) \neq 0$ in $D_{k-}$ with at least one zero on $T_{k}$, where $k \geq 1$, and all zeros of $s(z)$ lie in $T_{1} \cup D_{1-}$. Then, for $z \in T_{1}$,

$$
\left|r^{\prime}(s(z))\right| \leq \frac{1}{2 m m^{\prime}}\left[\left|B^{\prime}(z)\right|-\frac{m n(k-1) \cdot|r(s(z))|^{2}}{(1+k) \cdot\|r \circ s\|^{2}}\right] \cdot\|r \circ s\|,
$$

where $\quad m^{\prime}=\min _{z \in T_{1}}|s(z)|$. Equality holds for $r(s(z))=((z+k) /(z-a))^{m n} \quad$ where $s(z)=z^{m}$ and $B(z)=((1-a z) /(z-a))^{m n}, a>1$, and $k \geq 1$ at $z=1$.

When $k=1$ and $r(s(z))$ has a zero on $T_{k}$, Corollary 2 generalizes Theorem 5 .

\section{Data Availability}

No data were used to support this study.

\section{Conflicts of Interest}

The authors declare that there are no conflicts of interest regarding the publication of this paper.

\section{Acknowledgments}

This work has received a scholarship under the Research Fund for Supporting Lecturer to Admit High Potential Student to Study and Research on His Expert Program Year 2018 from the Graduate School, Khon Kaen University, Thailand (Grant no. 612JT217).

\section{References}

[1] S. Bernstein, Lecons sur les Propriétés extrémales et la meilleure approximation des fonctions analytiques d'une variable réelle, Gauthier Villars, Paris, France, 1926.
[2] P. D. Lax, "Proof of a conjecture of P. Erdös on the derivative of a polynomial," Bulletin of the American Mathematical Society, vol. 50, no. 8, pp. 509-513, 1944.

[3] P. Turán, "Über die Ableitung von polynomen," Compositio Mathematica, vol. 7, pp. 89-95, 1939.

[4] X. Li, R. N. Mohapatra, and R. S. Rodriguez, "Bernstein-type inequalities for rational functions with prescribed poles," Journal of the London Mathematical Society, vol. 51, no. 2, pp. 523-531, 1995.

[5] A. Aziz and W. M. Shah, "Some refinements of Bernstein-type inequalities for rational functions," Glasnik Matematicki, vol. 32, no. 52, pp. 29-37, 1997.

[6] A. Aziz and B. A. Zarger, "Some properties of rational functions with prescribed poles," Canadian Mathematical Bulletin, vol. 42, no. 4, pp. 417-426, 1999.

[7] N. Arunrat and K. M. Nakprasit, "Bounds of the derivative of some classes of rational functions," http://arxiv.org/abs/2001. 09791.

[8] I. Qasim and A. Liman, "Bernstein type inequalities for rational functions," Indian Journal of Pure and Applied Mathematics, vol. 46, no. 3, pp. 337-348, 2015.

[9] A. Aziz and Q. M. Dawood, "Inequalities for a polynomial and its derivative," Journal of Approximation Theory, vol. 54, no. 3, pp. 306-313, 1988. 